\title{
Article \\ Catenin Alpha 2 May Be a Biomarker or Potential Drug Target in Psychiatric Disorders with Perseverative Negative Thinking
}

\author{
Nora Eszlari ${ }^{1,2, *(\mathbb{D})}$, Zsolt Bagyura ${ }^{3} \mathbb{D}$, Andras Millinghoffer ${ }^{2,4,5}$, Tamas Nagy ${ }^{4} \mathbb{D}$, Gabriella Juhasz ${ }^{1,6,7}(\mathbb{D}$, \\ Peter Antal ${ }^{4,5}$, Bela Merkely ${ }^{3}$ and Gyorgy Bagdy 1,2,6
}

1 Department of Pharmacodynamics, Faculty of Pharmacy, Semmelweis University, Nagyvárad tér 4, H-1089 Budapest, Hungary; juhasz.gabriella@pharma.semmelweis-univ.hu (G.J.); bagdy.gyorgy@pharma.semmelweis-univ.hu (G.B.)

2 NAP-2-SE New Antidepressant Target Research Group, Hungarian Brain Research Program, Semmelweis University, Nagyvárad tér 4, H-1089 Budapest, Hungary; milli@mit.bme.hu

3 Heart and Vascular Center, Semmelweis University, Városmajor utca 68, H-1122 Budapest, Hungary; bagyura@zortal.hu (Z.B.); merkely.bela@med.semmelweis-univ.hu (B.M.)

4 Department of Measurement and Information Systems, Budapest University of Technology and Economics, Magyar Tudósok krt. 2, H-1521 Budapest, Hungary; tnagy@mit.bme.hu (T.N.); antal@mit.bme.hu (P.A.)

5 Abiomics Europe Ltd., Zólyomi út 23, H-1118 Budapest, Hungary

6 MTA-SE Neuropsychopharmacology and Neurochemistry Research Group, Hungarian Academy of Sciences, Semmelweis University, Nagyvárad tér 4, H-1089 Budapest, Hungary

7 SE-NAP 2 Genetic Brain Imaging Migraine Research Group, Hungarian Brain Research Program, Semmelweis University, Nagyvárad tér 4, H-1089 Budapest, Hungary

* Correspondence: eszlari.nora@pharma.semmelweis-univ.hu; Tel.: +36-14591500 (ext. 56153)

check for

updates

Citation: Eszlari, N.; Bagyura, Z.; Millinghoffer, A.; Nagy, T.; Juhasz, G.; Antal, P.; Merkely, B.; Bagdy, G. Catenin Alpha 2 May Be a Biomarker or Potential Drug Target in Psychiatric Disorders with Perseverative Negative Thinking. Pharmaceuticals 2021, 14, 850. https://doi.org/10.3390/ ph14090850

Academic Editor: Karolina Pytka

Received: 27 July 2021

Accepted: 19 August 2021

Published: 26 August 2021

Publisher's Note: MDPI stays neutral with regard to jurisdictional claims in published maps and institutional affiliations.

Copyright: (c) 2021 by the authors. Licensee MDPI, Basel, Switzerland. This article is an open access article distributed under the terms and conditions of the Creative Commons Attribution (CC BY) license (https:// creativecommons.org/licenses/by/ $4.0 /)$.

\begin{abstract}
AlphaN-catenin gene CTNNA2 has been implicated in intrauterine brain development, as well as in several psychiatric disorders and cardiovascular diseases. Our present aim was to investigate CTNNA2 gene-wide associations of single-nucleotide polymorphisms (SNPs) with psychiatric and cardiovascular risk factors to test the potential mediating role of rumination, a perseverative negative thinking phenotype in these associations. Linear mixed regression models were run by FaST-LMM within a sample of 795 individuals from the Budakalasz Health Examination Survey. The psychiatric outcome variables were rumination and its subtypes, and ten Brief Symptom Inventory (BSI) scores including, e.g., obsessive-compulsive, depression, anxiety, hostility, phobic anxiety, and paranoid ideation. Cardiovascular outcome variables were BMI and the Framingham risk scores for cardiovascular disease, coronary heart disease, myocardial infarction, and stroke. We found nominally significant CTNNA2 associations for every phenotype. Rumination totally mediated the associations of CTNNA2 rs17019243 with eight out of ten BSI scores, but none with Framingham scores or BMI. Our results suggest that CTNNA2 genetics may serve as biomarkers, and increasing the expression or function of CTNNA2 protein may be a potential new therapeutic approach in psychiatric disorders with perseverative negative thinking including, e.g., depression. Generally, an antiruminative agent could be a transdiagnostic and preventive psychopharmacon.
\end{abstract}

Keywords: CTNNA2; catenin alpha 2; rumination; perseverative cognition; Framingham Risk Score; body mass index; Brief Symptom Inventory; psychiatric symptoms

\section{Introduction}

The CTNNA2 gene and the encoded catenin (cadherin-associated protein) alpha 2 ( $\alpha \mathrm{N}$-catenin) protein have a crucial role in brain development. It has been suggested to regulate the branching of actin. Actin, in turn, guides the cytoskeleton's microtubules within growing neurites of developing and migrating neurons of the fetal cortex [1].

This risk gene acting at an early, intrauterine stage of brain development, has been associated with numerous psychiatric and psychological phenotypes in humans. It has been implicated in bipolar disorder [2], adult attention-deficit/hyperactivity disorder 
(ADHD) [3], alcoholism [4], schizophrenia, and general cognitive function [5], as well as in success in smoking cessation [6]. Moreover, it has shown associations with personality traits and endophenotypes that lie on possible pathways between genes and disorders, namely: impulsivity, which covers both novelty seeking and lack of planning [7], as well as a common visual endophenotype of schizophrenia and autism spectrum disorder, namely deficits in sensitivity to visual stimuli of a low spatial and high temporal frequency [8].

Although it has not survived multiple testing correction at a genome-wide level, it has shown a very significant association with Framingham Risk Score, an indicator of cardiovascular disease risk [9], and similarly with orthostatic hypotension [10].

It is a very challenging question whether or not the same mechanism of CTNNA2 acts in distinct pathways of psychiatric and cardiovascular risk. Regarding the potential psychological endophenotypes that mediate between genes and these distinct types of disorders, rumination would be a good candidate to investigate.

Rumination, or depressive rumination, is a stable response style to the person's own distress and depressed mood [11,12]. This trait involves a repetitive and passive focus, a fixation on personal problems and feelings, as well as on their causes and consequences, without taking action [11]. Therefore, this perseverative cognition prolongs mental representations, e.g., affective and physiological activation patterns of a stressor, being a moderator between stress and its health consequences [13].

Rumination has indeed been suggested as a transdiagnostic risk factor [14]. It predicts the future onset of major depression, even over many years [15]. It has been found to be present in all phases of bipolar disorder, associated with symptoms of depression, hypomania, and anxiety [16], and has been shown to have a higher level in social anxiety than in non-anxious subjects [17]. Rumination also predicted symptoms of post-traumatic stress disorder (PTSD) one year [18] and even three years [19] after a motor vehicle accident. It also predicted the future onset of substance abuse and binge eating in female adolescents [20], as well as the future level of alcohol use in alcohol abusers [21]. Rumination is also associated with premenstrual disorders [22] and shows its notable maladaptive aspects in schizophrenia as well [23].

Rumination, a form of a maintained "action preparation" stress response because of an affective, attentional, and autonomic inflexibility, has also been suggested to predict somatic complaints one year later [13]. There is also evidence that rumination is associated with a slower recovery of heart rate or blood pressure after stress, and such a slower cardiovascular recovery, in turn, predicts the future development of hypertension, even when controlling for the initial reactivity level $[13,24]$. A direct relationship between rumination and the future emergence of cardiovascular diseases, as well as the precedence of either cognitive or autonomic inflexibility in these relationships, still has to be elucidated [24].

Our present aim was to test the potential mediating role of rumination and its subtypes in CTNNA2-associated psychiatric and cardiovascular risk. For this, first, we tested associations of rumination with psychiatric and cardiovascular phenotypes. After that, we tested the role of gene-wide single-nucleotide polymorphisms (SNPs) of CTNNA2 in psychiatric symptoms, rumination, and cardiovascular risk factors, including body mass index (BMI) and Framingham scores. Finally, we tested the mediating role of rumination in CTNNA2-associated psychiatric and cardiovascular phenotypes.

\section{Results}

\subsection{Associations between Rumination Score and Disease Risk Phenotypes}

The mean, standard error, and standard deviation for each variable, as well as statistical power for the outcome variables and Cronbach's alpha values for psychometric scales, are presented in Table 1.

While rumination was associated with all ten Brief Symptom Inventory (BSI) scores, no cardiovascular risk factor was associated with it (Table 2). A moderate positive correlation was found in all cases. 
Table 1. Descriptive statistics for the measures of our study $(n=795)$. Range of Framingham scores can be between 0 and $100 \%$, BSI scores can range from 0 to 4 , and RRS scores from 1 to 4 . The results suggest that our study sample is generally overweighted and has an intermediate risk for CVD, but a low risk for specific CVD diseases. We can also see a wide range of statistical power values to detect CTNNA2 SNP effects on all phenotypes, and good internal consistencies for psychometric scales. BMI—body mass index; CVD—cardiovascular disease; CHD—coronary heart disease; HCHD—hard coronary heart disease: myocardial infarction or coronary death; BSI-Brief Symptom Inventory; RRS—Ruminative Response Scale.

\begin{tabular}{|c|c|c|c|c|c|}
\hline Variable & Mean & $\begin{array}{c}\text { Standard Error } \\
\text { of Mean }\end{array}$ & $\begin{array}{l}\text { Standard } \\
\text { Deviation }\end{array}$ & $\begin{array}{c}\text { Power to Detect } \\
\text { CTNNA2 SNP Effect }\end{array}$ & Cronbach's Alpha \\
\hline Age at medical examination & 53.72 & 0.490 & 13.815 & & \\
\hline Age at questionnaire filling & 54.85 & 0.500 & 14.109 & & \\
\hline BMI & 27.39 & 0.179 & 5.034 & $5.05-99.99 \%$ & \\
\hline Framingham-CVD & 13.78 & 0.426 & 11.998 & $5.01-76.98 \%$ & \\
\hline Framingham-CHD & 8.59 & 0.277 & 7.821 & $5.02-98.58 \%$ & \\
\hline Framingham-HCHD & 4.17 & 0.181 & 5.097 & $5.04-99.99 \%$ & \\
\hline Framingham-stroke & 2.83 & 0.117 & 3.288 & $5.11-99.99 \%$ & \\
\hline BSI global severity index & 0.55 & 0.018 & 0.508 & $9.58-99.99 \%$ & 0.957 \\
\hline BSI somatization & 0.51 & 0.022 & 0.617 & $8.08-99.99 \%$ & 0.800 \\
\hline BSI obsessive-compulsive & 0.70 & 0.025 & 0.703 & $7.37-99.99 \%$ & 0.828 \\
\hline BSI interpersonal sensitivity & 0.94 & 0.025 & 0.695 & $7.42-99.99 \%$ & 0.643 \\
\hline BSI depression & 0.48 & 0.021 & 0.606 & $8.20-99.99 \%$ & 0.862 \\
\hline BSI anxiety & 0.54 & 0.024 & 0.668 & $7.62-99.99 \%$ & 0.824 \\
\hline BSI hostility & 0.50 & 0.020 & 0.566 & $8.67-99.99 \%$ & 0.733 \\
\hline BSI phobic anxiety & 0.32 & 0.021 & 0.584 & $8.45-99.99 \%$ & 0.800 \\
\hline BSI paranoid ideation & 0.71 & 0.024 & 0.673 & $7.59-99.99 \%$ & 0.737 \\
\hline BSI psychoticism & 0.42 & 0.020 & 0.564 & $8.70-99.99 \%$ & 0.675 \\
\hline RRS rumination & 1.89 & 0.018 & 0.503 & $9.67-99.99 \%$ & 0.812 \\
\hline RRS brooding & 1.91 & 0.020 & 0.570 & $8.62-99.99 \%$ & 0.759 \\
\hline RRS reflection & 1.86 & 0.021 & 0.602 & $8.24-99.99 \%$ & 0.753 \\
\hline
\end{tabular}

Table 2. Pearson correlations of RRS score residuals with cardiovascular and psychiatric risk scores $(n=795)$. Significant $(p \leq 0.05)$ correlations are marked in bold. Among the cardiovascular risk scores, only BMI is correlated, and only weakly, with RRS scores: positively with brooding and negatively with reflection. RRS rumination and brooding are correlated moderately with all ten BSI scores, but reflection has a weak correlation with obsessive-compulsive and depressive symptoms. $p$ — $p$-value; BMI—body mass index; CVD—cardiovascular disease; CHD—coronary heart disease; HCHD—hard coronary heart disease: myocardial infarction or coronary death; BSI—Brief Symptom Inventory; RRS—Ruminative Response Scale.

\begin{tabular}{|c|c|c|c|c|}
\hline Variabl & & $\begin{array}{l}\text { RRS Rumination } \\
\text { Score Residual }\end{array}$ & $\begin{array}{l}\text { RRS Brooding } \\
\text { Score Residual }\end{array}$ & $\begin{array}{l}\text { RRS Reflection } \\
\text { Score Residual }\end{array}$ \\
\hline \multirow{2}{*}{ RRS rumination score residual } & Pearson correlation & 1 & 0.508 & 0.532 \\
\hline & $p$ & & $2.148 \times 10^{-53}$ & $2.291 \times 10^{-59}$ \\
\hline \multirow{2}{*}{ RRS brooding score residual } & Pearson correlation & 0.508 & 1 & -0.459 \\
\hline & $p$ & $2.148 \times 10^{-53}$ & & $1.263 \times 10^{-42}$ \\
\hline \multirow{2}{*}{ RRS reflection score residual } & Pearson correlation & 0.532 & -0.459 & 1 \\
\hline & $p$ & $2.291 \times 10^{-59}$ & $1.263 \times 10^{-42}$ & \\
\hline \multirow{2}{*}{ BMI } & Pearson correlation & -0.017 & 0.079 & -0.095 \\
\hline & $p$ & 0.632 & 0.026 & 0.007 \\
\hline \multirow{2}{*}{ Framingham-CVD } & Pearson correlation & 0.019 & 0.020 & -0.001 \\
\hline & $p$ & 0.598 & 0.565 & 0.982 \\
\hline \multirow{2}{*}{ Framingham-CHD } & Pearson correlation & 0.015 & 0.011 & 0.005 \\
\hline & $p$ & 0.667 & 0.756 & 0.890 \\
\hline \multirow{2}{*}{ Framingham-HCHD } & Pearson correlation & 0.027 & 0.017 & 0.011 \\
\hline & $p$ & 0.451 & 0.636 & 0.754 \\
\hline
\end{tabular}


Table 2. Cont.

\begin{tabular}{|c|c|c|c|c|}
\hline \multicolumn{2}{|c|}{ Variable } & $\begin{array}{l}\text { RRS Rumination } \\
\text { Score Residual }\end{array}$ & $\begin{array}{l}\text { RRS Brooding } \\
\text { Score Residual }\end{array}$ & $\begin{array}{l}\text { RRS Reflection } \\
\text { Score Residual }\end{array}$ \\
\hline \multirow{2}{*}{ Framingham-stroke } & Pearson correlation & 0.024 & 0.020 & 0.005 \\
\hline & $p$ & 0.502 & 0.568 & 0.896 \\
\hline \multirow{2}{*}{ BSI global severity index } & Pearson correlation & 0.454 & 0.435 & 0.040 \\
\hline & $p$ & $1.357 \times 10^{-41}$ & $4.553 \times 10^{-38}$ & 0.258 \\
\hline \multirow{2}{*}{ BSI somatization } & Pearson correlation & 0.278 & 0.299 & -0.007 \\
\hline & $p$ & $1.586 \times 10^{-15}$ & $7.339 \times 10^{-18}$ & 0.835 \\
\hline \multirow{2}{*}{ BSI obsessive-compulsive } & Pearson correlation & 0.364 & 0.301 & 0.080 \\
\hline & $p$ & $2.272 \times 10^{-26}$ & $4.043 \times 10^{-18}$ & 0.024 \\
\hline \multirow{2}{*}{ BSI interpersonal sensitivity } & Pearson correlation & 0.355 & 0.398 & -0.026 \\
\hline & $p$ & $5.627 \times 10^{-25}$ & $1.259 \times 10^{-31}$ & 0.471 \\
\hline \multirow{2}{*}{ BSI depression } & Pearson correlation & 0.456 & 0.403 & 0.074 \\
\hline & $p$ & $4.600 \times 10^{-42}$ & $1.834 \times 10^{-32}$ & 0.037 \\
\hline \multirow{2}{*}{ BSI anxiety } & Pearson correlation & 0.404 & 0.384 & 0.039 \\
\hline & $p$ & $1.355 \times 10^{-32}$ & $2.087 \times 10^{-29}$ & 0.271 \\
\hline \multirow{2}{*}{ BSI hostility } & Pearson correlation & 0.334 & 0.343 & 0.007 \\
\hline & $p$ & $4.033 \times 10^{-22}$ & $2.014 \times 10^{-23}$ & 0.852 \\
\hline \multirow{2}{*}{ BSI phobic anxiety } & Pearson correlation & 0.302 & 0.276 & 0.040 \\
\hline & $p$ & $2.952 \times 10^{-18}$ & $2.199 \times 10^{-15}$ & 0.256 \\
\hline \multirow{2}{*}{ BSI paranoid ideation } & Pearson correlation & 0.363 & 0.395 & -0.013 \\
\hline & $p$ & $3.710 \times 10^{-26}$ & $5.287 \times 10^{-31}$ & 0.707 \\
\hline \multirow{2}{*}{ BSI psychoticism } & Pearson correlation & 0.368 & 0.346 & 0.040 \\
\hline & $p$ & $6.039 \times 10^{-27}$ & $8.015 \times 10^{-24}$ & 0.266 \\
\hline
\end{tabular}

Associations between Other Ruminative Response Scale (RRS) Scores Brooding and Reflection and Disease Risk Phenotypes

RRS rumination has two subtypes: brooding and reflection [25]. Brooding is a "moody pondering", passive, and maladaptive comparison of a person's current situation with some unachieved standard. In contrast, reflection is a more adaptive subtype, which means a neutrally valenced, purposeful turning inward to engage in cognitive problem solving and thus to alleviate the depressed mood.

The RRS score residuals significantly correlated with each other - both brooding and reflection had a positive correlation with rumination, and had a negative correlation with each other. While RRS brooding had a moderate positive association with all ten BSI scores, only BMI among cardiovascular risk factors was associated with brooding-weakly and positively. RRS reflection had weak significant associations, was positive with depressive and obsessive-compulsive symptoms, and was negative with BMI (Table 2).

\subsection{Associations of CTNNA2 SNPs with Each of the Phenotypes, in FaST-LMM Regression Models}

Figure 1 demonstrates that while no SNP within CTNNA2 or its buffer region extended by \pm 10 kilobase survived the Bonferroni-corrected significance threshold, many SNPs showed a nominal association with every phenotype. Rs12613937 and rs13030077 were the two most significant SNPs for Framingham-CVD, Framingham-CHD, and Framingham-HCHD. 

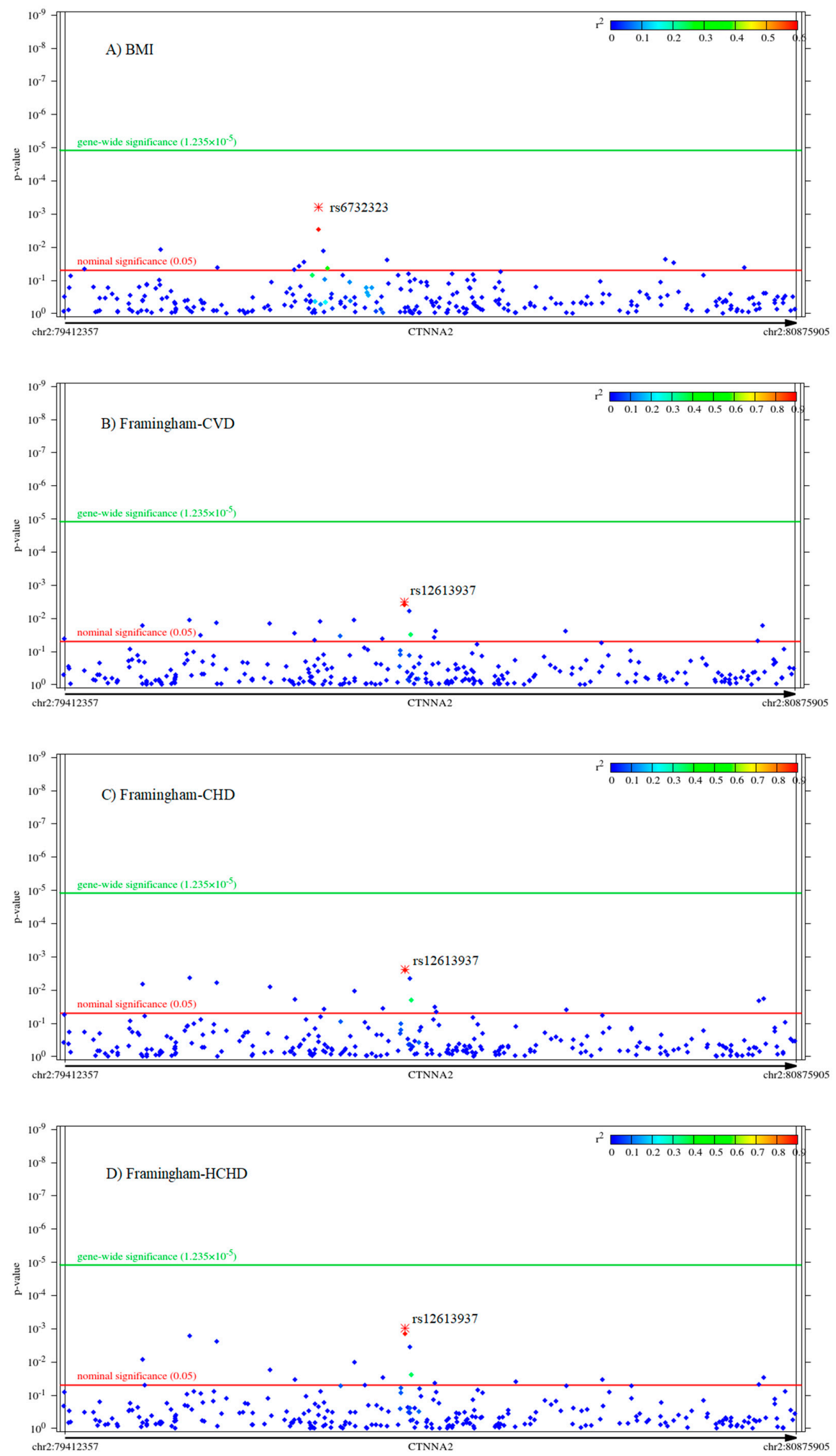

Figure 1. Cont. 

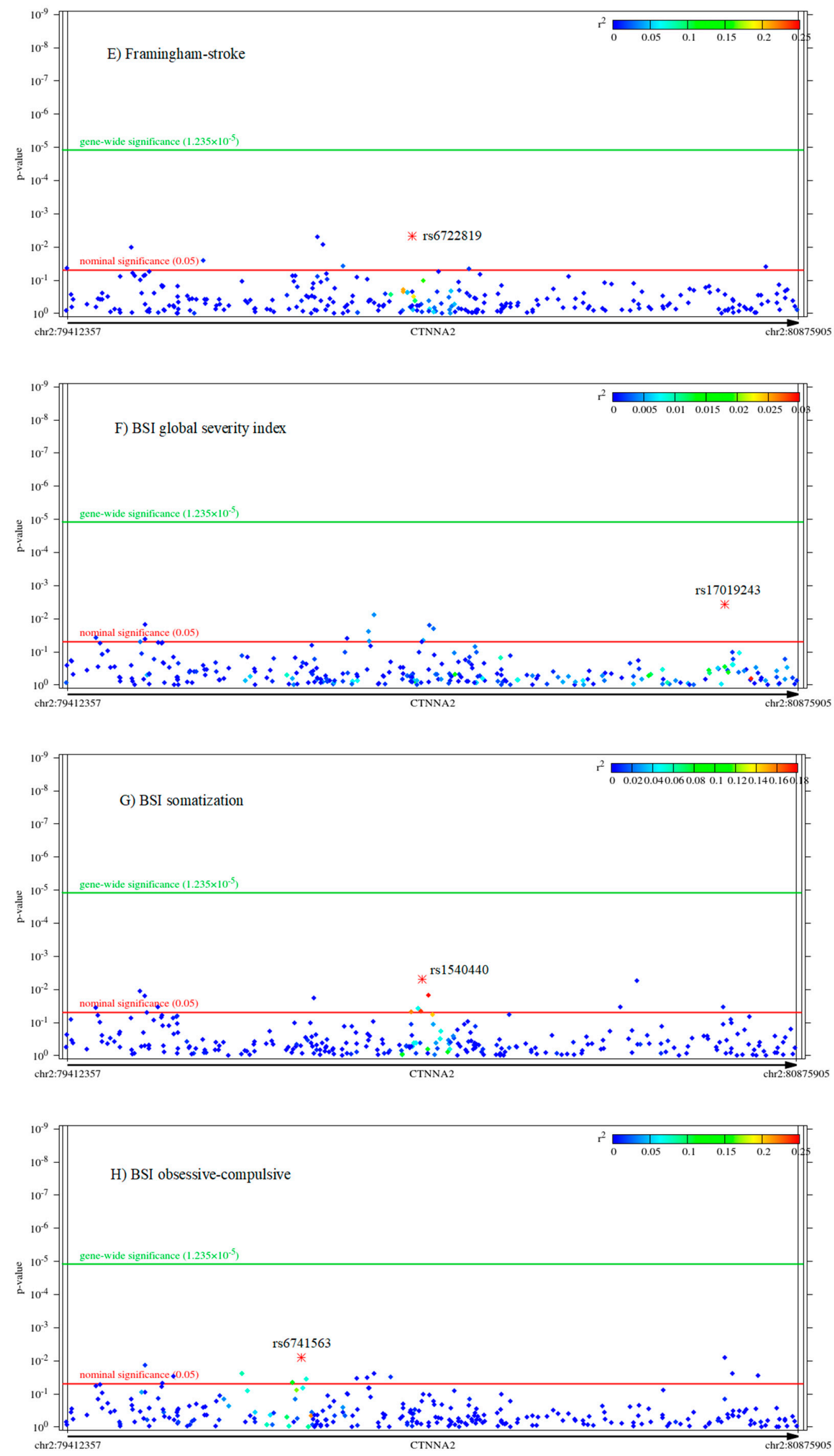

Figure 1. Cont. 

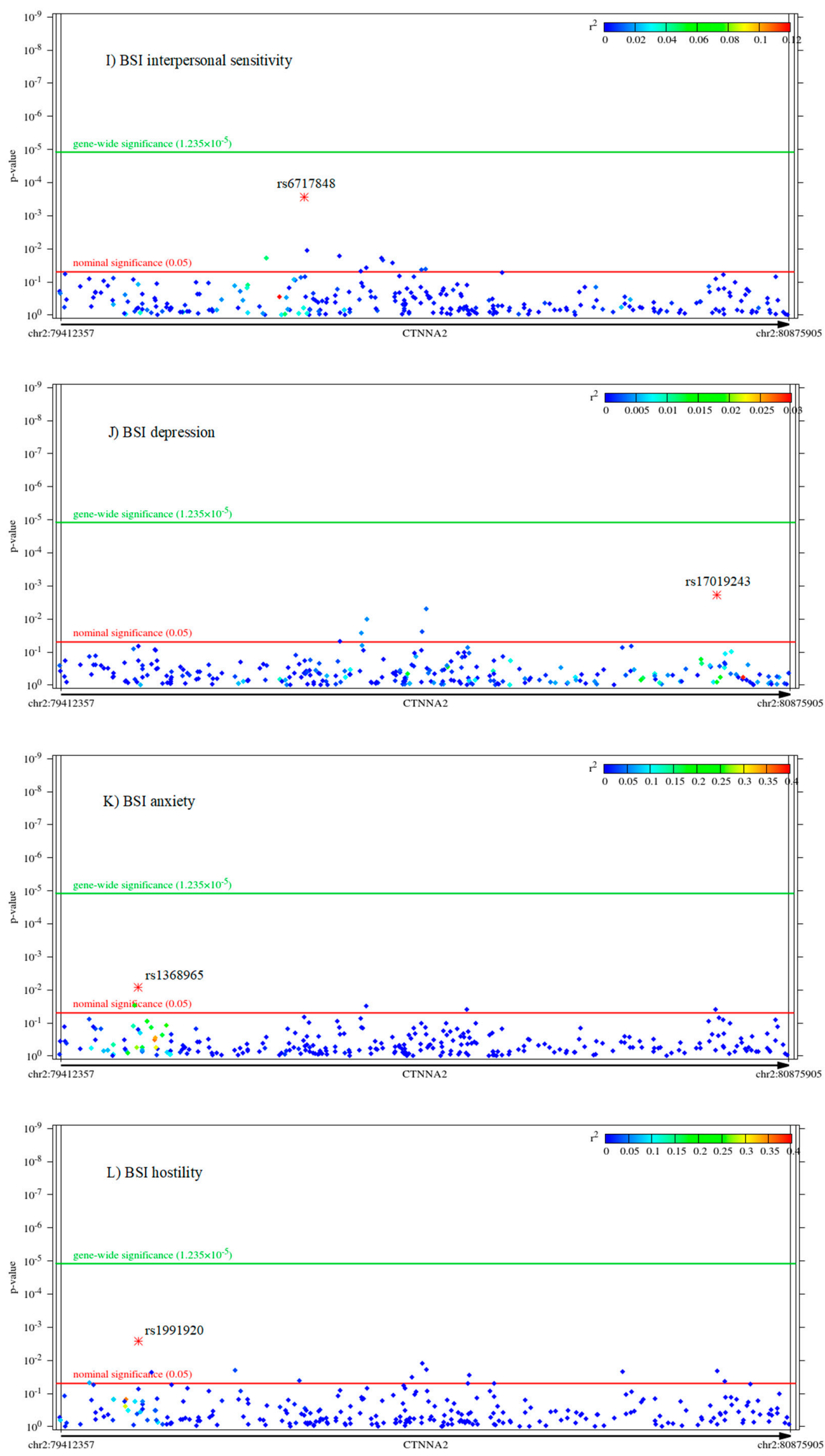

Figure 1. Cont. 

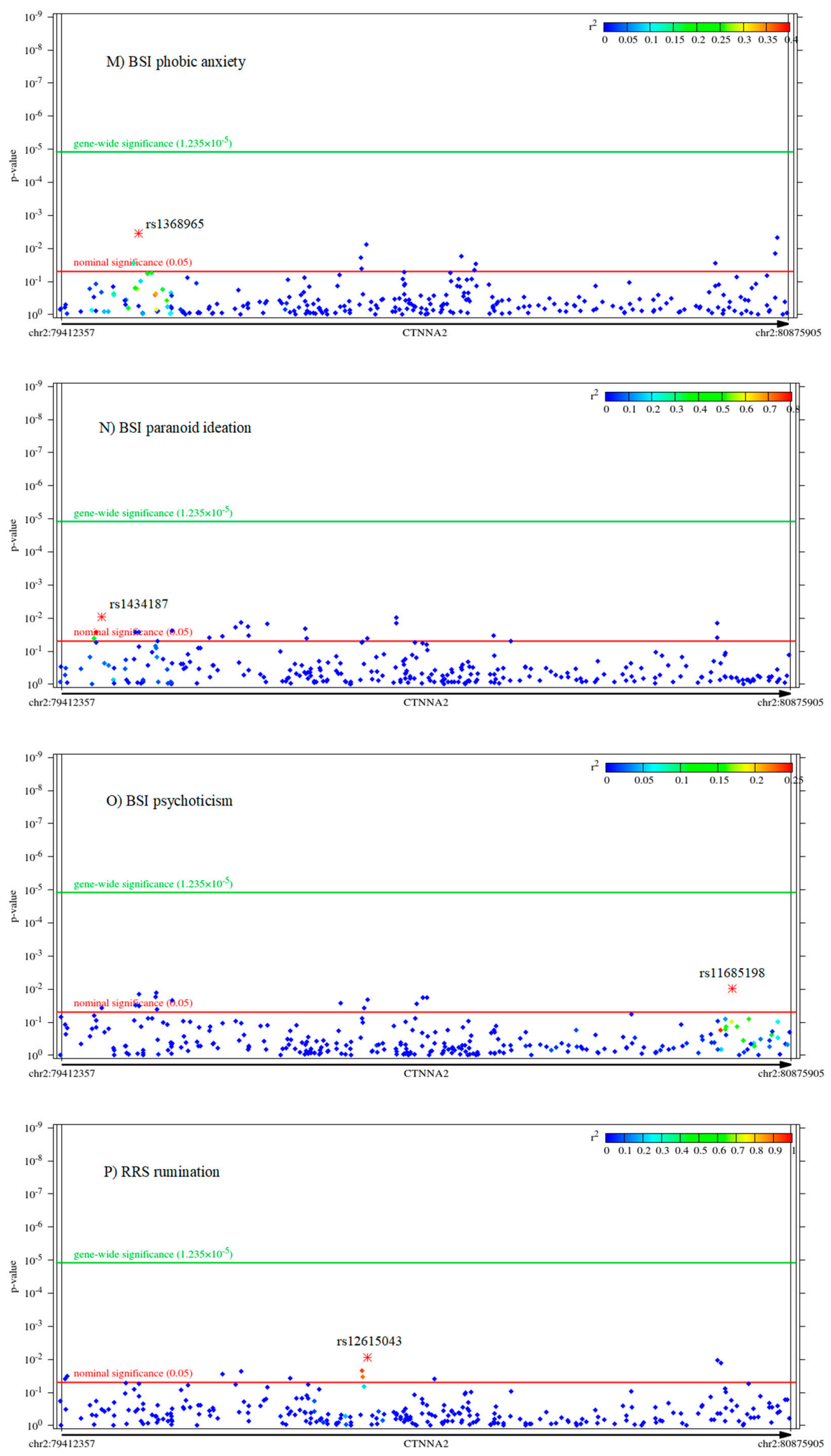

Figure 1. Cont. 

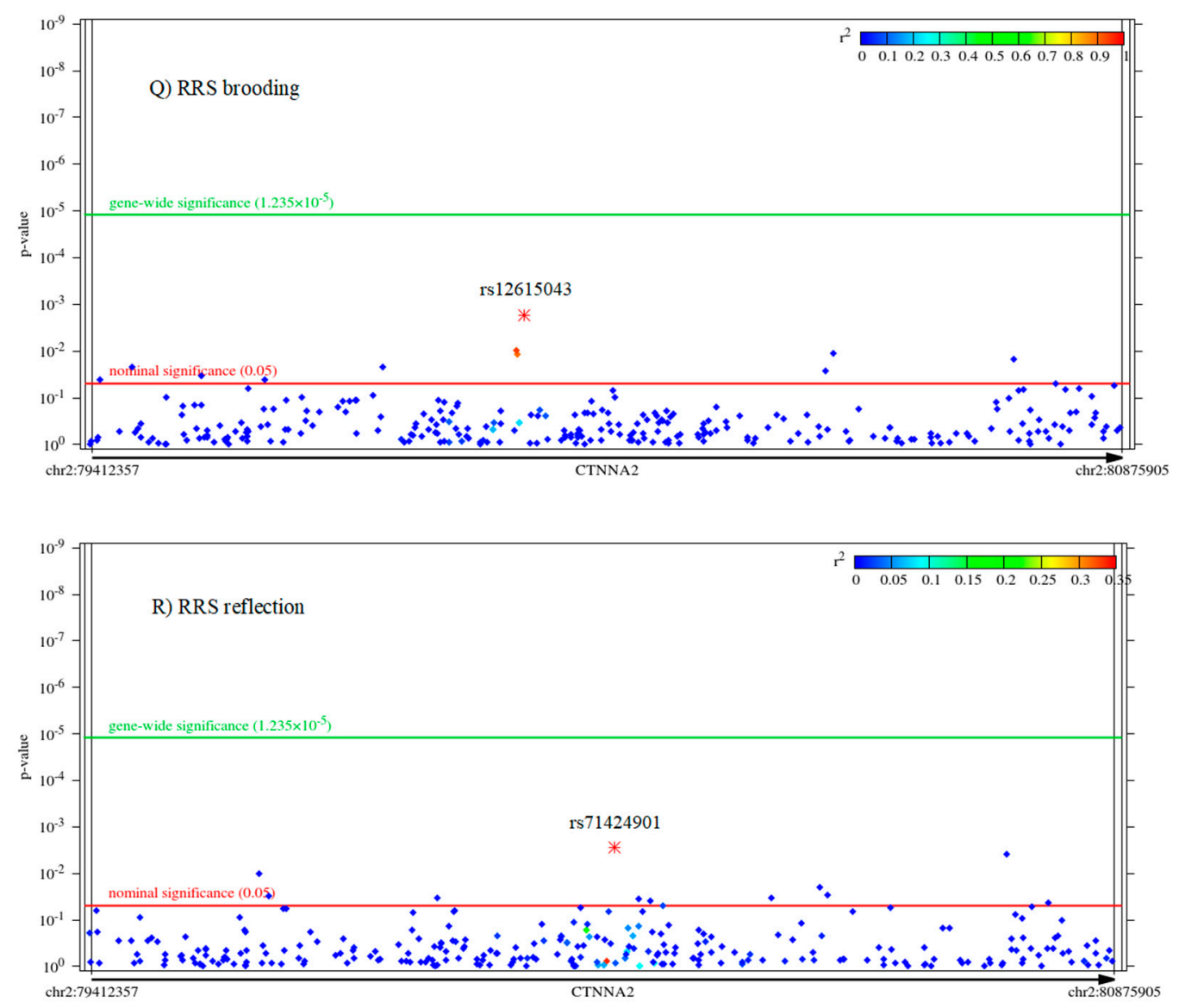

Figure 1. Manhattan plots for the associations of each CTNNA2 SNP with each outcome variable. (A-E) display cardiovascular risk phenotypes, (F-O) display BSI psychiatric symptom scores, and (P-R) display RRS rumination, brooding and reflection scores, respectively. The $p$-value is displayed with two thresholds, Bonferroni-corrected gene-wide and nominal significance, as a function of the SNP position within the gene. We can see that, while no SNP survived the gene-wide significant threshold, there are nominally significant associations for all 18 phenotypes. $\mathrm{R}^{2}$-linkage disequilibrium with the most significant SNP (marked with asterisk and given by name); SNP—single-nucleotide polymorphism; chr—chromosome; BMI—body mass index; CVD—cardiovascular disease; CHD—coronary heart disease; HCHD—hard coronary heart disease: myocardial infarction or coronary death; BSI—Brief Symptom Inventory; RRS—Ruminative Response Scale.

\subsection{The Mediating Role of Rumination between CTNNA2 and Psychiatric Symptoms}

Mediation testing has two prerequisites. First, the potential mediator has to be significantly associated with the outcome variable (Table 2), and second, the potential mediator and the outcome have to share at least one significant SNP with each other (Supplementary Table S1).

As none of the cardiovascular risk scores are correlated with rumination (Table 2), no mediation is possible. Rumination has positive correlations with all ten BSI scores (Table 2) and shares six significant SNPs with at least one of the BSI scores (Supplementary Table S1). Consequently, these six SNPs, rumination, and the respective BSI scores meet both the prerequisites of mediation testing. Among the shared SNPs with rumination, associations with eight BSI scores were found with two SNPs: rs12615043 and rs17019243 (Supplementary Table S1). In detail, rs12615043 is significantly associated with rumination, as well as with BSI global severity index (GSI), obsessive-compulsive, interpersonal sensitivity, depression, anxiety, phobic anxiety, paranoid ideation, and psychoticism scores. Rs17019243 is significantly associated with rumination, BSI GSI, somatization, obsessive-compulsive, depression, anxiety, hostility, phobic anxiety, and paranoid ideation scores. 
To show the potential mediating role of rumination in BSI associations with the six shared SNPs, we ran secondary analyses in FaST-LMM regression models for the ten BSI scores, covarying the rumination score (Supplementary Table S2). Most of the SNP-BSI score associations lost their significance if covarying rumination, suggesting a mediating role.

As they had associations with most of the BSI scales, rs12615043 and rs17019243 were included in a Bayesian Multilevel Analysis (BMLA) with all of the 18 investigated phenotypes, plus sex and age, to reveal their relevance regarding RRS and BSI scores within the network of all of the investigated variables. Because of the low sample sizes within the separate genotype groups (rs12615043 GG: 5; GA: 83; AA: 550; rs17019243 TT: 5; TC: 52; CC: 577), a dominant model was used, with binary encoding. The results pointed out that while rs12615043 proved to be irrelevant regarding either the RRS or BSI scores, rs17019243 had high posterior probabilities of a relationship with RRS rumination and brooding, BSI somatization, and the categories of both RRS and BSI (Figure 2 and Supplementary Table S3). Supported by the results of this non-frequentist statistical approach, in addition to the results of LMM regression models, rs17019243 was selected for testing mediation via rumination towards BSI scores, where primary analyses showed associations, namely GSI, somatization, obsessive-compulsive, depression, anxiety, hostility, phobic anxiety, and paranoid ideation scores.

Statistical mediation was investigated by a structural equation model (SEM) analysis including 634 unrelated participants who had non-missing data on rs17019243, sex, age, all three RRS scores, and all ten BSI scores. Rs17019243 was included according to a dominant model-whether or not the participant carried the minor $\mathrm{T}$ allele. The following regressions were included to control for the effects of confounding variables. The eight BSI scores and the potential mediator RRS rumination score were regressed on sex and age. Potential genetic stratification of the population also had to be corrected for, so as to avoid false positive genetic associations with the investigated phenotypes. As the first principal component (PC) of the genome showed a correlation with the BSI global severity index and anxiety, and the 10th PC of the genome showed a correlation with BSI obsessive-compulsive and hostility scores-these BSI scores were regressed on the respective PC in the model.

The model showed excellent fit indices (Figure 3). To display the direct effects of rs17019243 for rumination and BSI scores within the model, regression (path) coefficients were standardized by standard deviation of the outcome variable (Figure 3). Similarly, to show the mediating role of rumination within the same model, estimates of the indirect effects were standardized by standard deviations of the outcome variable (Table 3). The results revealed that while rs 17019243 had no significant direct effect on any of the psychiatric symptom scores if a mediated effect via rumination was also included in the model, all of these mediated effects were significant (Figure 3 and Table 3). These results are in line with the notion that CTNNA2 SNP effects on the BSI scores are entirely mediated by rumination. 


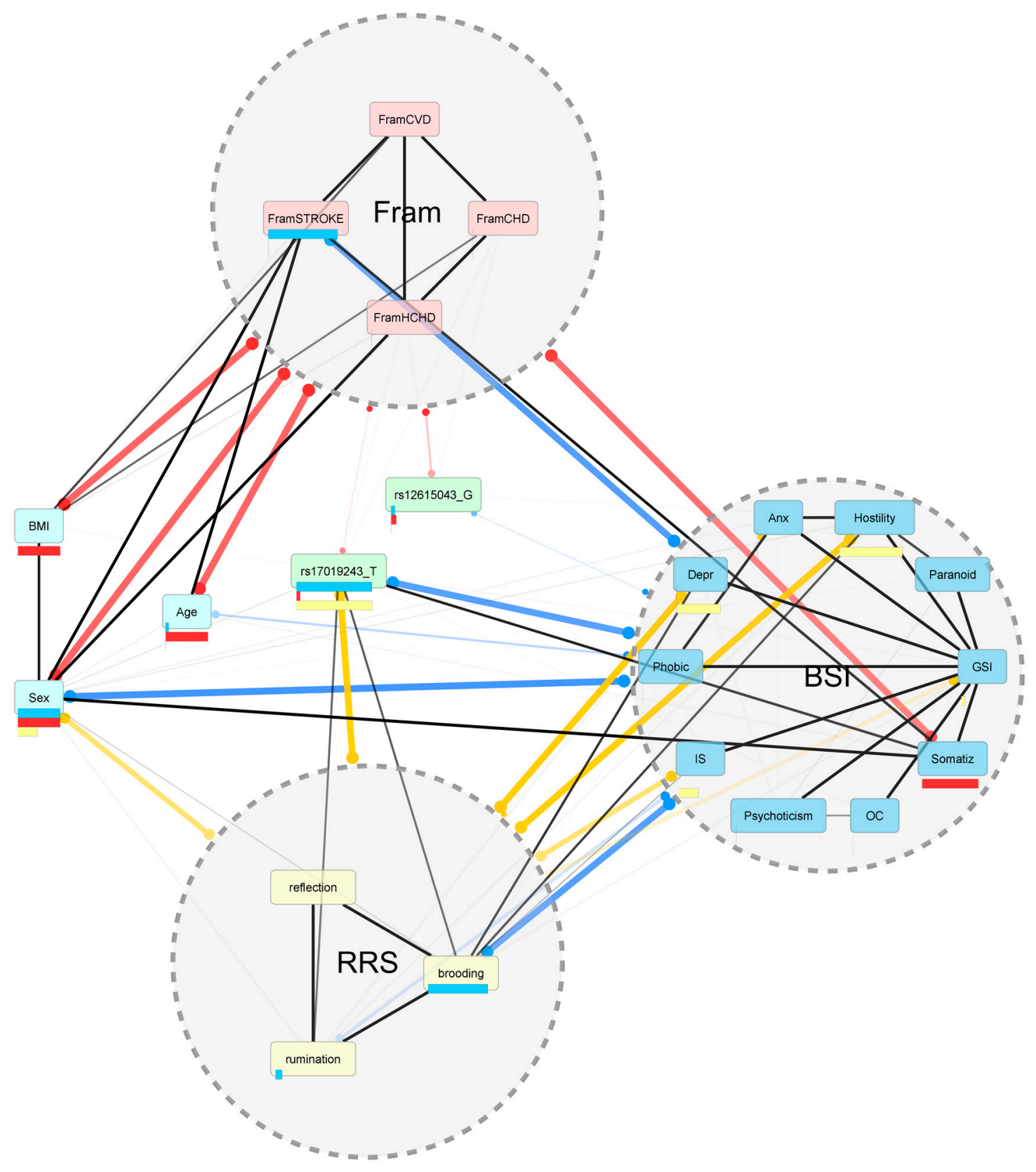

Figure 2. The resulting network model from the Bayesian Multilevel Analysis. The edges (all lines) represent a probabilistic relationship between the variables, namely single variables and categories of variables. Single variables include the 18 phenotypes, sex, age, and the two SNPs. Categories of variables include Framingham, RRS, and BSI. The thickness of the edges is proportional with the posterior probability, signifying a more probable real-life connection between the variables (numerical posterior probability values are detailed in Supplementary Table S3). The black edges connect single variables with each other, and the colored edges connect single variables with the categories, where the color encoding is as follows: red-Framingham; yellow-RRS; blue-BSI. The length of the horizontal bars below the nodes represents the posterior probability values with respect to the categories. The results suggest a real-life relationship between rs17019243 and RRS, and BSI single variables and categories as well. However, rs12615043 has no real-life relationship with any of them. RRS—Ruminative Response Scale; BSI—Brief Symptom Inventory; BMI—body mass index; CVD—cardiovascular disease; $\mathrm{CHD}$ — coronary heart disease; HCHD—hard coronary heart disease: myocardial infarction or coronary death; GSI—global severity index; anx—anxiety; depr—depression; IS—interpersonal sensitivity; OC—obsessive-compulsive. 


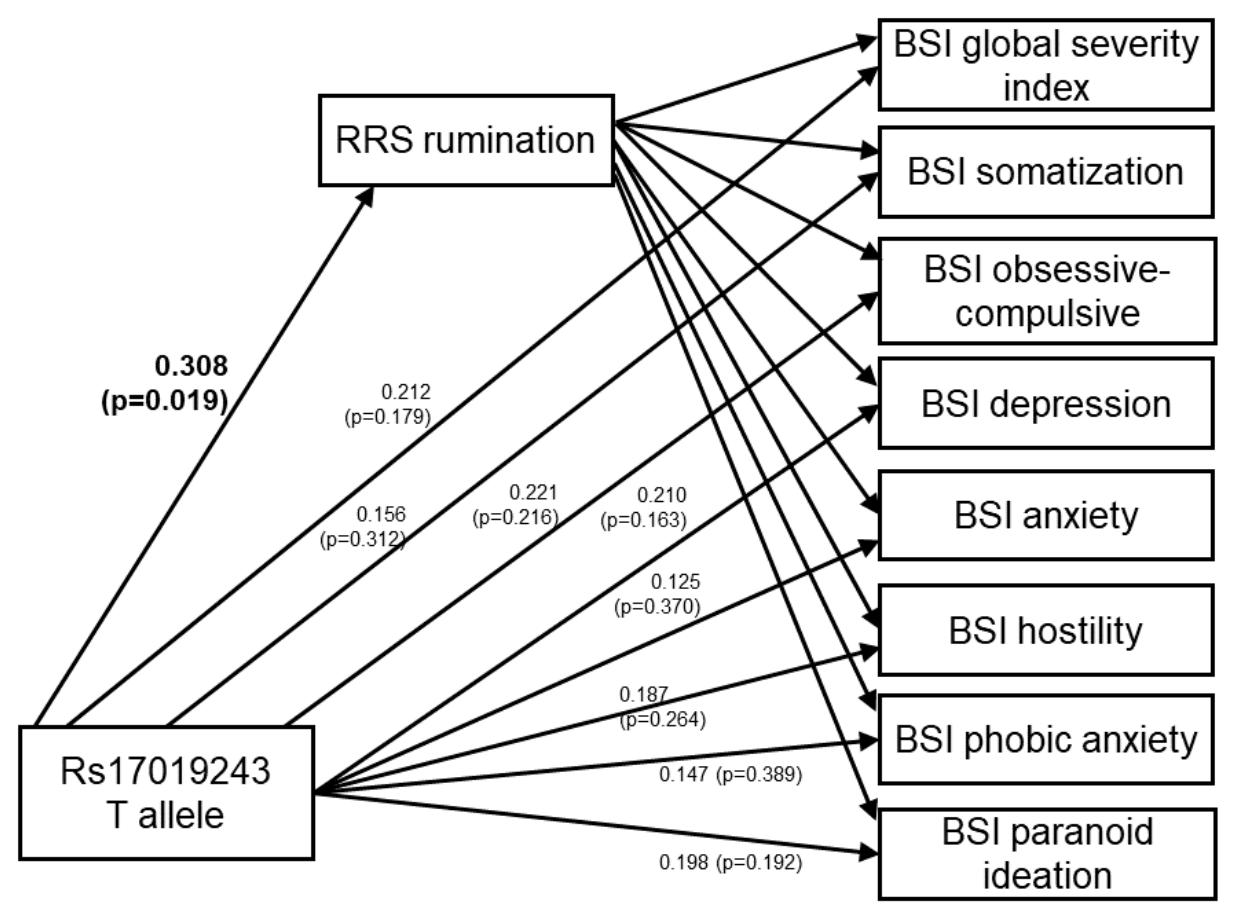

Figure 3. Structural equation modeling to test the direct and indirect effects of CTNNA2 rs17019243 for BSI psychiatric symptom scores via RRS rumination within the same model. Model fit indices: $\chi^{2}=20.184(\mathrm{df}=14 ; p=0.1244) ; \mathrm{RMSEA}=0.026(\mathrm{PCLOSE}=0.949) ; \mathrm{CFI}=0.999 ; \mathrm{TLI}=0.992$. Direct effects of rs17019243 are displayed in the figure, and the significant $(p \leq 0.05)$ one is marked in bold. The results suggest that rs17019243 has a direct effect on RRS rumination, but has no direct effect on any of the BSI scores. BSI-Brief Symptom Inventory; RRS—Ruminative Response Scale; df-degrees of freedom; RMSEA—root mean squared error of approximation; PCLOSE—significance of the statistical hypothesis that RMSEA is different from the desirable $\leq 0.05$; CFI-comparative fit index ( $>0.90$ indicates a good model fit, and $>0.95$ indicates a very good model fit); TLI-Tucker-Lewis index ( $>0.90$ indicates a good, and $>0.95$ a very good model fit).

Table 3. Standardized estimates of the indirect effects of CTNNA2 rs17019243 on each BSI score via RRS rumination. All mediated effects are significant at a $p \leq 0.05$. We can see that rs17019243 acts on eight BSI scores via RRS rumination, with the strongest effect on GSI, depression, anxiety, obsessive-compulsive, and paranoid ideation scores. RRS-Ruminative Response Scale; BSI-Brief Symptom Inventory; GSI—global severity index.

\begin{tabular}{ccccccccc}
\hline \multicolumn{7}{c}{ CTNNA2 rs17019243 $\rightarrow$ RRS Rumination $\rightarrow$ BSI Score } \\
\hline GSI & Somatization & $\begin{array}{c}\text { Obsessive- } \\
\text { Compulsive }\end{array}$ & Depression & Anxiety & Hostility & $\begin{array}{c}\text { Phobic } \\
\text { Anxiety }\end{array}$ & $\begin{array}{c}\text { Paranoid } \\
\text { Ideation }\end{array}$ \\
\hline 0.146 & 0.098 & 0.117 & 0.146 & 0.133 & 0.107 & 0.098 & 0.114 \\
$(p=0.020)$ & $(p=0.023)$ & $(p=0.020)$ & $(p=0.021)$ & $(p=0.020)$ & $(p=0.022)$ & $(p=0.022)$ & $(p=0.023)$ \\
\hline
\end{tabular}

The Mediating Role of Other RRS Scores Brooding and Reflection between CTNNA2 and Psychiatric Symptoms

The results of the brooding and reflection are shown in the same tables as the rumination (Table 2 and Supplementary Tables S1 and S2).

As none of the Framingham scores were correlated with any of the RRS scores (Table 2), no mediation was possible. Regarding BMI, although it has a weak association with both brooding and reflection scores (Table 2), it shares no significant SNP with any of them (Supplementary Table S1), excluding the possibility of mediation. Similarly, in case of RRS reflection, although it was correlated with both BSI obsessive-compulsive and depressive symptoms, it shared no significant SNP with any of them, excluding any mediating 
role. Regarding associations and positive correlations with BSI scores and the number of shared SNPs, brooding was very similar to rumination (Table 2 and Supplementary Tables S1 and S2). However, among the rs12615043 and rs17019243, brooding had a significant association only with rs12615043 according to LMM primary analyses (Supplementary Table S1), which SNP, in turn, did not fulfill the criterion of relevance in BMLA results (Figure 2 and Supplementary Table S3), therefore no mediating role of brooding was tested.

\section{Discussion}

Our study was the first to investigate whether SNPs covering the whole CTNNA2 gene are related to a diverse set of psychiatric and cardiovascular risk scores, as well as whether these associations are mediated by a transdiagnostic repetitive negative thinking phenotype-rumination. Our results demonstrated that while CTNNA2 SNPs had nominal associations with all psychiatric and cardiovascular risk scores, rumination mediated CTNNA2's role only towards psychiatric, but not cardiovascular risk scores.

\subsection{CTNNA2 Has Pleiotropic Effects on Cardiovascular Phenotypes and Rumination}

CTNNA2 SNPs showed nominal associations with each of the investigated 18 phenotypes. However, while CTNNA2 associations with psychiatric risk scores seem to be mediated by rumination, its associations with cardiovascular risk scores seemed independent of rumination.

Although rumination, as an inflexibly maintained stress response and a repetitive thought style, seemed to be a relevant cardiovascular risk $[13,24]$, it was not a significant factor in the CTNNA2 variant-associated increase of Framingham risk scores.

In fact, CTNNA2 was found to have associations with BMI and Framingham scores independently of rumination. CTNNA2 SNPs may act on cardiovascular phenotypes through brain mechanisms other than rumination, as it has been hypothesized in case of CTNNA2 involvement in orthostatic hypotension [10].

Another possible explanation is that the CTNNA2 protein is present in the heart, and thus has a direct cardiovascular effect. Although, according to the Human Protein Atlas v20.0 [26], CTNNA2 shows the highest RNA and protein expression levels in brain, male tissues, and granulocytes (https:/ / www.proteinatlas.org/ENSG00000066032-CTNNA2 / tissue accessed on: 22 November 2020), it also has some low expression values in different cell types of the heart muscle (https: / www.proteinatlas.org/ENSG00000066032-CTNNA2 / celltype accessed on 22 November 2020).

Further studies are needed to elucidate the precise nature of this pleiotropy on rumination and risks for coronary heart disease, stroke, cardiovascular diseases generally, or BMI.

\subsection{CTNNA2 Effects on Divergent Psychiatric Symptoms Are Entirely Mediated by Rumination}

Our results revealed that while CTNNA2 rs17019243 acted on eight BSI scores via RRS rumination, no direct effect could be detected from the SNP towards any of the BSI scores.

Although the genetic background of rumination has been extensively investigated in previous studies [27-29], only a few studies have considered the possible endophenotypic nature of rumination, namely its putative causal role on the pathways between specific genes and specific disorders. These few studies, implicating the roles of synaptic plasticity candidate genes BDNF [30] and CREB1 [31], as well as serotonin receptor gene HTR2A [28] and folate pathway gene MTHFD1L [32], have exclusively focused on depression as a relevant disorder, or on depressive symptoms. No other disorders have been studied as the end of pathways from certain genes through rumination. Nevertheless, a twin study demonstrated a substantial genetic overlap between rumination and depression, though a more complicated picture in the association of rumination and eating pathology, and a firm role of environmental influences instead of genetics in the association of rumination and dependence vulnerability [33]. The BSI questionnaire of our present study did not assess eating pathology or addiction, thus we could not investigate the endophenotypic 
features of RRS scales between CTNNA2 and these two kinds of psychopathology. However, our results suggested the endophenotypic relevance of rumination in pathways between CTNNA2 and several other psychiatric symptom scores, also corroborating former results with depressive symptoms. Furthermore, the transdiagnostic nature of rumination in CTNNA2-associated psychiatric disorders cannot be separated or restricted to brooding or reflection subtypes.

\subsection{Catenin Alpha 2 Protein (Encoded by CTNNA2) as a Potential Drug Target in Multiple Psychiatric Disorders or Multimorbid Conditions}

Although having a prominent role in intrauterine stages, CTNNA2 is also expressed in the adult brain, showing different levels in patient groups versus controls, underpinning its potential as a drug target for psychiatric disorders.

CTNNA2 has shown a reduced postmortem brain expression in schizophrenic patients versus controls, investigated within the parvocellular region of the mediodorsal thalamic nucleus [34], which has connections with several cortical and sub-cortical structures [35]. Moreover, schizophrenic non-smokers had a reduced hippocampal CTNNA2 expression post mortem compared with schizophrenic smokers or mentally healthy non-smokers [36].

Catenin alpha 2 protein (encoded by CTNNA2 gene in humans and Ctnna2 in mice) is a linker between cadherin adhesion receptors and the actin cytoskeleton, and mice lacking its gene showed abnormally motile dendritic spine heads in the synapses of hippocampal neurons [37]. Its overexpression in these cells, however, restored the normal morphology of dendritic spines, and stabilized it over time [37], suggesting a possible therapeutic use in genetically vulnerable subgroups.

Pointing to the behavioral correlates and the possibility of a successful intervention in the case of a genetic vulnerability, the deletion of a gene partly overlapping Ctnna2 in mice resulted in a larger startle to noise, an impaired pavlovian conditioned fear, and a weaker pre-pulse inhibition, all of which could be rescued by expressing Ctnna 2 in such mice [38].

Although the human adult expression of CTNNA2 has been investigated only in schizophrenia, and pre-pulse inhibition is an endophenotype of schizophrenia [39], our results extend the relevance of CTNNA2 in a diverse set of psychiatric symptoms and also in a transdiagnostic endophenotype-rumination.

Thus, in general, increasing the expression of CTNNA2 or increasing the function of its catenin alpha 2 protein may have therapeutic potential in several psychiatric symptoms and perseverative negative thinking.

\subsection{Limitations}

Our study has several significant limitations. First, our cross-sectional design and the uncovered statistical mediation could only suggest the endophenotypic nature of rumination between CTNNA2 and several psychiatric symptoms, but a longitudinal design would be needed to prove its causal role. Former studies on the possible endophenotypic role of rumination have also been cross-sectional; therefore, a longitudinal study would be crucial.

Second, our sample size entailed a very limited power to detect the effects of rare alleles or of weak effect sizes (Table 1). In our power calculations, the minimum minor allele frequency (MAF) was 0.01 , and the hypothesized minimum effect size was $\beta=0.08$. In case of all outcome variables, a larger sample would be needed to detect these kinds of effects. However, rs17019243 had a MAF of only 0.0489 in the sample of our mediation analyses, and had a standardized regression coefficient of 0.308 on RRS rumination in the model, suggesting enough power to detect effects in the case of not minimal but low MAFs and effect sizes. 


\section{Materials and Methods}

\subsection{Participants}

This study is part of the Budakalasz Health Examination Survey (BHES), a crosssectional voluntary cardiovascular screening program [40]. BHES was performed in 20112013 targeting the adult population (>20 years, around 8000 inhabitants) of a CentralHungarian town (Budakalasz). The participation rate in the Budakalasz Health Examination Survey was approximately $30 \%(n=2420)$ of the total eligible population.

Our present study included BHES participants with quality-controlled genomic data and non-missing phenotypic data on sex, age, rumination, and the investigated cardiovascular and psychiatric risk phenotypes (see below, in Section 4.4).

The study was approved by the Medical Research Council Scientific and Ethics Committee (approval no. 8224-0/2011/EKU [265/PI/11]). All of the procedures were done in accordance with the ethical standards of the responsible committee on human experimentation (institutional and national) and the Helsinki Declaration of 1975, as revised in 2000 (5). Written informed consent was obtained from all participants.

\subsection{Measures}

Medical history with special attention to cardiovascular disease (CVD)-related signs and symptoms, as well as lifestyle, was recorded by an experienced physician. Medical history was regarded as positive for hypertension if documented. For previously unknown hypertension, the following cut-off points were used: $>140 \mathrm{mmHg}$ systolic and/or $>90 \mathrm{mmHg}$ diastolic blood pressure. Current and former regular smokers were both considered smokers.

Anthropometric parameters (height and weight), rounded to the nearest $0.1 \mathrm{~cm}$ and $0.1 \mathrm{~kg}$, were measured in a standing position while participants were wearing light indoor clothing without shoes. BMI was calculated with the Quatelet's form [41]. Routine laboratory tests were performed in our Institution's Central Laboratory with rigorous quality control. The concentration of lipid fractions was measured by using a colorimetric assay (Roche Diagnostics Ltd., Mannheim, Germany).

The ten years risk for developing CVD, coronary heart disease (CHD), myocardial infarction or coronary death (hard coronary heart disease (HCHD)), and stroke incidences were calculated for each participant using the relevant Framingham equations [42-44]. In detail, the Framingham-CVD score indicates a risk for any cardiovascular disease, and includes age, diabetes, smoking status, treated and untreated systolic blood pressure, total cholesterol, and high-density lipoprotein cholesterol (HDL-C) categories. FraminghamCHD indicates a risk score for coronary heart disease, and consists of gender, age, diabetes, smoking, blood pressure, total cholesterol categories, and low-density lipoprotein cholesterol (LDL-C) categories. Framingham-HCHD comprises age, total cholesterol, HDL-C, systolic blood pressure, treatment for hypertension, and smoking status. Framinghamstroke risk score includes age, systolic blood pressure, diabetes mellitus, cigarette smoking, prior CVD, atrial fibrillation, left ventricular hypertrophy, and use of hypertensive medication. The range of Framingham scores can be between 0 and $100 \%$. In general, individuals with a low risk have $10 \%$ or less risk at 10 years, with intermediate risk of $10-20 \%$, and with high risk of $20 \%$ or more.

To assess a wide range of psychiatric symptoms, the 53-item Brief Symptom Inventory (BSI) was used [45]. In addition to the global severity index (GSI) comprising all of the items, BSI has nine specific scales. These are somatization, obsessive-compulsive, interpersonal sensitivity, depression, anxiety, hostility, phobic anxiety, paranoid ideation, and psychoticism. Answers could be rated on a five-point Likert scale (0-4) for each item. Additional items that had not loaded straightforwardly on any factor in the original exploratory study were added to the depression scale in our study, because of their content.

Rumination was measured by the 10-item Ruminative Response Scale (RRS) [25], rated on a four-point Likert scale. Five items assessed the maladaptive brooding subtype, and the other five items belonged to the more adaptive reflection subtype. 
In the case of each of the ten BSI scales and the three RRS scales, the sum score was divided by the number of items the participant responded to on that scale, and this mean score was used in all of the analyses.

Age was registered as the difference between birth date and the date of either medical examination or questionnaire filling, in years, rounded to one decimal.

\subsection{Genotyping and Quality Control}

Genotyping was performed using the Axiom Precision Medicine Research Array chip (https:/ / www.thermofisher.com/order/catalog/product/902981\#/902981 accessed on: 16 February 2021). Raw genotyping results were filtered using a rigorous quality control process, which followed the protocol in [46] and consisted of the following steps. First, biallelic, single-nucleotide variants with a minor-allele frequency (MAF) not less than 0.01 were retained. Then, variants and samples with a missing rate greater than 0.01 were excluded. This step was performed in an iterative manner, gradually increasing the missingness boundary from 0.1 through 0.05 to 0.01 , at each step filtering genotypes first, and then the samples. Then, the genotypes not in Hardy-Weinberg equilibrium were discarded (i.e., the ones for which Plink's "-hwe" test yielded a $p$-value less than $10^{-5}$ ). The SNPs remaining after this last step were kept for analysis, however the samples were further filtered.

For this latter part, the first a set of independent SNPs was selected using Plink's "-indep-pairwise" command, using a sliding window of 1500 variants with a step size of 150 variants and with a linkage disequilibrium (LD) boundary of $R^{2} \leq 0.2$. The samples for which the calculated heterozygosity F-score differs from the population-level mean by more than 3.0 standard deviations were excluded.

For analyses that require unrelated individuals, an identical-by-descent (IBD) filtering, with a threshold of 0.1875 and keeping only one member of the closely related pairs, was applied before the final heterozygosity-based filtering step.

This QC process resulted 274 SNPs within a \pm 10 kilobase extended boundaries of CTNNA2 gene, according to genome build GRCh37/hg19.

\subsection{Analyses}

Descriptive statistics and associations between the phenotype measures were calculated using IBM SPSS v25 for the sample of 795 individuals from the primary analyses (see below). This sample consisted of 317 males and 478 females. To control for the variance of confounding factors, standardized residuals were used for association testing between the phenotypes, as follows. RRS rumination was regressed on sex and age. RRS brooding was regressed on sex, age, and RRS reflection. Finally, RRS reflection was regressed on sex, age, and RRS brooding. All three linear regression models were calculated with an enter method. The standardized residuals of each model were then tested for Pearson correlation with the five cardiovascular risk scores and the ten BSI scores.

For the primary analyses, a linear mixed modeling (LMM) approach, FaST-LMM [47], was used for a sample of 795 participants who had genetic data and non-missing phenotypic data for sex, age, BMI, Framingham scores, and BSI and RRS scores. The LMM with each SNP was run in an additive model for each of BMI, the four Framingham scores, the ten BSI scores, and the three RRS scores as the outcome variable. All of the models covaried sex, and a kinship matrix was included to handle the relatedness among participants and population stratification. This kinship matrix was calculated using the LOCO (leaveone-chromosome-out) method, meaning that kinship was estimated based on the whole genomic data, except for chromosome 2 (where CTNNA2 resides). Age was also a covariate in each model, registered at the time point of either the medical examination (in case of BMI and Framingham scores) or the questionnaire filling (in case of BSI and RRS scores). For the RRS subscales of brooding and reflection, the other subscale was included as an additional covariate. Accounting for the LD between SNPs, we considered 225 independent (effective) SNPs behind our 274 SNPs, according to Gao et al.'s method [48]. The 18 models 
with these 225 effective SNPs resulted in 4050 tests and, consequently, a $1.235 \times 10^{-5}$ Bonferroni-corrected significance threshold.

Power analyses for these primary tests were performed with Quanto v1.2 [49]. The means and standard deviations used for the calculations are detailed in Table 1 for each outcome variable. For every outcome variable, the type I error rate was set to a two-sided 0.05 , and the minor allele frequency (MAF) range was hypothesized between 0.01 and 0.5 by 0.01 . Based on previous studies, the effect size was expected to be $\beta=0.08$ [9] or $\beta=1.62$ [7] in an additive model, for every variable. The range of potential MAF values and the two hypothesized effect sizes resulted in a range of statistical power values to detect the CTNNA2 SNP effects.

Secondary analyses were aimed at testing the mediating role of rumination in the significant findings of the primary analyses. For this purpose, we selected SNPs fulfilling the criteria of showing nominally significant $(p \leq 0.05)$ associations with both an RRS score and a cardiovascular or psychiatric risk score that had been significantly related to that particular RRS score according to the descriptive statistics. First, LMM was run with each of these SNPs for the particular cardiovascular or psychiatric risk score as an outcome, with the same method as in primary analyses, but including the particular RRS score as an additional covariate.

Second, from these SNPs shared between an RRS score and a disease risk score, we selected those that had been significantly associated with most of the disease risk scores and at least one RRS score in the primary analyses. To further filter these SNPs according to the statistical methods other than separate regression equations for each outcome, we performed a Bayesian Multilevel Analysis (BMLA), which treated all relationships between all variables in one complex model. For the BMLA, continuous phenotypic variables were discretized by their terciles, and we used a sample of unrelated individuals $(n=629)$ who had non-missing data on all of the included variables. Within the BMLA, a Markov Chain Monte Carlo method was used to estimate the a posteriori probabilities of the edges between the single variables in the Bayesian network with 1,000,000 burnin steps and 5,000,000 sampling steps [50,51]. Using the same method, the categorical posterior probabilities were calculated for the BSI, RRS, and Framingham categories, which incorporated the corresponding variables. We used the Cooper-Herskovits priors [52] and the maximum number of parent connections were set to 4 . Sex, age, and the selected SNPs were treated as exogenous variables, meaning that they could fulfill only causal roles in the model. Note that an a posteriori probability signifies the chance that a given relationship is present between the variables; thus, for example, a value above 0.5 means that a connection between variables is more probable than not.

SNPs showing strong a posteriori probabilities for both the particular RRS variables and the particular disease variables were selected for mediation testing.

Mplus v7.4 (https:/ / www.statmodel.com/ accessed on: 14 July 2020) was used to test the statistical mediation of the particular RRS score between the SNP and the risk scores in structural equation modeling (SEM), which also hypothesize direct and indirect effects in the same model. An MLR (maximum likelihood estimation with robust standard errors) method was used within a sample of unrelated individuals $(n=638)$ having genetic data and non-missing phenotypic data on sex, age, RRS scores, and all the risk scores investigated in these secondary analyses. As, in Mplus, we could not enter a genomewide kinship matrix to correct for relatedness and population stratification, only unrelated individuals were included, and the top ten principal components (PCs) of the genome were calculated to correct for potential stratification of the population. A PC was included in the SEM model if correlated with an outcome variable.

\section{Conclusions}

Our study suggests that the identified SNP of the CTNNA2 gene is associated with rumination, and this type of perseverative negative thinking mediates a wide variety of psychopathologies and psychiatric disorders. These data strongly underscore the neuro- 
biological basis of psychiatric disorders and point to the necessity of a new classification system of psychiatric disorders that was strongly suggested, but finally dropped at the construction of DSM-5.

Our data suggest the biomarker role of this gene, particularly its rs17019243 SNP, acting most likely through the CTNNA2 expression levels, for rumination independently of the psychiatric diagnosis. Consequently, our results may help to develop new perspectives within personalized medicine and the stratification of highly heterogeneous patient groups into more biologically homogeneous subgroups.

Furthermore, increasing the expression or function of the catenin alpha 2 protein may be a new therapeutic approach in psychiatric patients, especially with rumination in their background.

Another new, more general approach could be the development of a possible new antiruminative agent that could serve as a psychiatric diagnosis independent (transpsychopharmacon) medicine in psychiatry. As rumination may precede depression and PTSD, the treatment of rumination may also serve as a preventive medication in depression, schizophrenia, and other psychiatric disorders in this specific ruminating population.

Supplementary Materials: The following are available online at https:/ /www.mdpi.com/article/10 $.3390 / \mathrm{ph} 14090850 / \mathrm{s} 1$. Supplementary Tables S1-S3. Supplementary Table S1: Position and $p$-value of SNPs that proved to be nominally significant for any RRS score in primary analyses (Figure 1P-R). Supplementary Table S2: Results of secondary analyses in FaST-LMM regression models for the ten BSI scores, covarying either RRS rumination or brooding score. Supplementary Table S3: Posterior probabilities of a real-life relationship between each pair of single variables and categories of variables in a network model, calculated by a Bayesian Multilevel Analysis.

Author Contributions: Conceptualization, B.M., G.B., N.E. and G.J.; methodology, N.E., A.M., T.N. and P.A.; software, Z.B., A.M., T.N. and P.A.; validation, N.E., T.N. and P.A.; formal analysis, N.E., Z.B., A.M. and T.N.; investigation, Z.B. and N.E.; resources, Z.B., G.J., P.A., B.M. and G.B.; data curation, Z.B. and A.M.; writing-original draft preparation, N.E. and G.B.; writing—review and editing, N.E., Z.B., A.M., T.N., G.J., P.A. and G.B.; visualization, N.E., T.N. and A.M.; supervision, B.M. and G.B.; project administration, B.M. and G.B.; funding acquisition, B.M., G.B., G.J., P.A., Z.B. and N.E. All authors have read and agreed to the published version of the manuscript.

Funding: The study was supported by the Hungarian Brain Research Program (grants KTIA_13_NAPAII/14 and 2017-1.2.1-NKP-2017-00002); the National Development Agency (grant KTIA_NAP_13-12013-0001); the Hungarian Academy of Sciences, Hungarian National Development Agency, Semmelweis University, and the Hungarian Brain Research Program (grant KTIA_NAP_13-2-2015-0001, MTA-SE-NAP B Genetic Brain Imaging Migraine Research Group); the Hungarian Academy of Sciences (MTA-SE Neuropsychopharmacology and Neurochemistry Research Group); OTKA 119866; the NRDI Fund (TKP2020 IES, grant No. BME-IE-BIO), based on the charter of bolster issued by the NRDI Office under the auspices of the Ministry for Innovation and Technology; the New National Excellence Program of the Ministry for Innovation and Technology from the source of the National Research, Development and Innovation Fund (ÚNKP-20-4-II-SE-9 and ÚNKP-21-4-II-SE-1); the National Research, Development, and Innovation Office, Hungary (2019-2.1.7-ERA-NET-2020-00005), under the frame of ERA PerMed (ERAPERMED2019-108); the Thematic Excellence Programme (Tématerületi Kiválósági Program, 2020-4.1.1.-TKP2020) of the Ministry for Innovation and Technology in Hungary, within the framework of the Neurology and Translational Biotechnology thematic programmes of the Semmelweis University; the National Research, Development and Innovation Office of Hungary (NKFIA; NVKP-16-1-2016-0017); and the Higher Education Institutional Excellence Programme of the Ministry of Human Capacities in Hungary, within the framework of the Therapeutic Development thematic programme of the Semmelweis University.

Institutional Review Board Statement: The study was conducted according to the guidelines of the Declaration of Helsinki, and was approved by the Medical Research Council Scientific and Ethics Committee (approval no: 8224-0/2011/EKU (265/PI/11)).

Informed Consent Statement: Informed consent was obtained from all subjects involved in the study. 
Data Availability Statement: The data presented in this study are available upon request from the corresponding author. The data are not publicly available due to ethical considerations.

Acknowledgments: We would like to thank to Budakalasz's Health Club for their help in participant recruitment.

Conflicts of Interest: The authors declare no conflict of interest. The funders had no role in the design of the study; in the collection, analyses, or interpretation of data; in the writing of the manuscript; or in the decision to publish the results.

\section{References}

1. Schaffer, A.E.; Breuss, M.W.; Caglayan, A.O.; Al-Sanaa, N.; Al-Abdulwahed, H.Y.; Kaymakcalan, H.; Yilmaz, C.; Zaki, M.S.; Rosti, R.O.; Copeland, B.; et al. Biallelic loss of human CTNNA2, encoding alphaN-catenin, leads to ARP2/3 complex overactivity and disordered cortical neuronal migration. Nat. Genet. 2018, 50, 1093-1101. [CrossRef]

2. Scott, L.J.; Muglia, P.; Kong, X.Q.; Guan, W.; Flickinger, M.; Upmanyu, R.; Tozzi, F.; Li, J.Z.; Burmeister, M.; Absher, D.; et al. Genome-wide association and meta-analysis of bipolar disorder in individuals of European ancestry. Proc. Natl. Acad. Sci. USA 2009, 106, 7501-7506. [CrossRef]

3. Lesch, K.P.; Timmesfeld, N.; Renner, T.J.; Halperin, R.; Roser, C.; Nguyen, T.T.; Craig, D.W.; Romanos, J.; Heine, M.; Meyer, J.; et al. Molecular genetics of adult ADHD: Converging evidence from genome-wide association and extended pedigree linkage studies. J. Neural Transm. 2008, 115, 1573-1585. [CrossRef]

4. Song, C.; Zhang, H. TARV: Tree-based analysis of rare variants identifying risk modifying variants in CTNNA2 and CNTNAP2 for alcohol addiction. Genet. Epidemiol. 2014, 38, 552-559. [CrossRef]

5. Smeland, O.B.; Frei, O.; Kauppi, K.; Hill, W.D.; Li, W.; Wang, Y.; Krull, F.; Bettella, F.; Eriksen, J.A.; Witoelar, A.; et al. Identification of Genetic Loci Jointly Influencing Schizophrenia Risk and the Cognitive Traits of Verbal-Numerical Reasoning, Reaction Time, and General Cognitive Function. JAMA Psychiatry 2017, 74, 1065-1075. [CrossRef]

6. Uhl, G.R.; Liu, Q.R.; Drgon, T.; Johnson, C.; Walther, D.; Rose, J.E.; David, S.P.; Niaura, R.; Lerman, C. Molecular genetics of successful smoking cessation: Convergent genome-wide association study results. Arch. Gen. Psychiatry 2008, 65, 683-693. [CrossRef] [PubMed]

7. Ehlers, C.L.; Gizer, I.R.; Bizon, C.; Slutske, W.; Peng, Q.; Schork, N.J.; Wilhelmsen, K.C. Single nucleotide polymorphisms in the REG-CTNNA2 region of chromosome 2 and NEIL3 associated with impulsivity in a Native American sample. Genes Brain Behav. 2016, 15, 568-577. [CrossRef] [PubMed]

8. Goodbourn, P.T.; Bosten, J.M.; Bargary, G.; Hogg, R.E.; Lawrance-Owen, A.J.; Mollon, J.D. Variants in the 1q21 risk region are associated with a visual endophenotype of autism and schizophrenia. Genes Brain Behav. 2014, 13, 144-151. [CrossRef]

9. Simonson, M.A.; Wills, A.G.; Keller, M.C.; McQueen, M.B. Recent methods for polygenic analysis of genome-wide data implicate an important effect of common variants on cardiovascular disease risk. BMC Med. Genet. 2011, 12, 146. [CrossRef] [PubMed]

10. Hong, K.W.; Kim, S.S.; Kim, Y. Genome-wide association study of orthostatic hypotension and supine-standing blood pressure changes in two korean populations. Genom. Inform. 2013, 11, 129-134. [CrossRef]

11. Nolen-Hoeksema, S.; Wisco, B.E.; Lyubomirsky, S. Rethinking Rumination. Perspect. Psychol. Sci. 2008, 3, 400-424. [CrossRef] [PubMed]

12. Smith, J.M.; Alloy, L.B. A roadmap to rumination: A review of the definition, assessment, and conceptualization of this multifaceted construct. Clin. Psychol. Rev. 2009, 29, 116-128. [CrossRef] [PubMed]

13. Brosschot, J.F.; Gerin, W.; Thayer, J.F. The perseverative cognition hypothesis: A review of worry, prolonged stress-related physiological activation, and health. J. Psychosom. Res. 2006, 60, 113-124. [CrossRef]

14. Nolen-Hoeksema, S.; Watkins, E.R. A Heuristic for Developing Transdiagnostic Models of Psychopathology: Explaining Multifinality and Divergent Trajectories. Perspect. Psychol. Sci. 2011, 6, 589-609. [CrossRef]

15. Aldao, A.; Nolen-Hoeksema, S.; Schweizer, S. Emotion-regulation strategies across psychopathology: A meta-analytic review. Clin. Psychol. Rev. 2010, 30, 217-237. [CrossRef]

16. Silveira, E.d.M., Jr.; Anna, M.K.-S., Jr. Rumination in bipolar disorder: A systematic review. Braz. J. Psychiatry 2015, 37, 256-263. [CrossRef] [PubMed]

17. Mellings, T.M.B.; Alden, L.E. Cognitive processes in social anxiety: The effects of self-focus, rumination and anticipatory processing. Behav. Res. Ther. 2000, 38, 243-257. [CrossRef]

18. Ehlers, A.; Mayou, R.A.; Bryant, B. Psychological predictors of chronic posttraumatic stress disorder after motor vehicle accidents. J. Abnorm. Psychol. 1998, 107, 508-519. [CrossRef] [PubMed]

19. Mayou, R.A.; Ehlers, A.; Bryant, B. Posttraumatic stress disorder after motor vehicle accidents: 3-year follow-up of a prospective longitudinal study. Behav. Res. Ther. 2002, 40, 665-675. [CrossRef]

20. Nolen-Hoeksema, S.; Stice, E.; Wade, E.; Bohon, C. Reciprocal relations between rumination and bulimic, substance abuse, and depressive symptoms in female adolescents. J. Abnorm. Psychol. 2007, 116, 198-207. [CrossRef]

21. Caselli, G.; Ferretti, C.; Leoni, M.; Rebecchi, D.; Rovetto, F.; Spada, M.M. Rumination as a predictor of drinking behaviour in alcohol abusers: A prospective study. Addiction 2010, 105, 1041-1048. [CrossRef] 
22. Craner, J.R.; Sigmon, S.T.; Martinson, A.A.; McGillicuddy, M.L. Premenstrual disorders and rumination. J. Clin. Psychol. 2014, 70, 32-47. [CrossRef] [PubMed]

23. Maurage, P.; Philippot, P.; Grynberg, D.; Leleux, D.; Delatte, B.; Mangelinckx, C.; Belge, J.B.; Constant, E. Imbalance between abstract and concrete repetitive thinking modes in schizophrenia. Compr. Psychiatry 2017, 78, 61-66. [CrossRef] [PubMed]

24. Larsen, B.A.; Christenfeld, N.J. Cardiovascular disease and psychiatric comorbidity: The potential role of perseverative cognition. Cardiovasc. Psychiatry. Neurol. 2009, 2009, 791017. [CrossRef]

25. Treynor, W.; Gonzalez, R.; Nolen-Hoeksema, S. Rumination reconsidered: A psychometric analysis. Cognit. Ther. Res. 2003, 27, 247-259. [CrossRef]

26. Uhlén, M.; Fagerberg, L.; Hallström, B.M.; Lindskog, C.; Oksvold, P.; Mardinoglu, A.; Sivertsson, Å.; Kampf, C.; Sjöstedt, E.; Asplund, A.; et al. Proteomics. Tissue-based map of the human proteome. Science 2015, 347, 1260419. [CrossRef] [PubMed]

27. Eszlari, N.; Millinghoffer, A.; Petschner, P.; Gonda, X.; Baksa, D.; Pulay, A.J.; Rethelyi, J.M.; Breen, G.; Deakin, J.F.W.; Antal, P.; et al. Genome-wide association analysis reveals KCTD12 and miR-383-binding genes in the background of rumination. Transl. Psychiatry 2019, 9, 019-0454. [CrossRef] [PubMed]

28. Eszlari, N.; Petschner, P.; Gonda, X.; Baksa, D.; Elliott, R.; Anderson, I.M.; Deakin, J.F.W.; Bagdy, G.; Juhasz, G. Childhood Adversity Moderates the Effects of HTR2A Epigenetic Regulatory Polymorphisms on Rumination. Front. Psychiatry 2019, 10, 394. [CrossRef]

29. Shaw, Z.A.; Hilt, L.M.; Starr, L.R. The developmental origins of ruminative response style: An integrative review. Clin. Psychol. Rev. 2019, 74, 101780. [CrossRef]

30. Hilt, L.M.; Sander, L.C.; Nolen-Hoeksema, S.; Simen, A.A. The BDNF Val66Met polymorphism predicts rumination and depression differently in young adolescent girls and their mothers. Neurosci. Lett. 2007, 429, 12-16. [CrossRef]

31. Juhasz, G.; Dunham, J.S.; McKie, S.; Thomas, E.; Downey, D.; Chase, D.; Lloyd-Williams, K.; Toth, Z.G.; Platt, H.; Mekli, K.; et al. The CREB1-BDNF-NTRK2 Pathway in Depression: Multiple Gene-Cognition-Environment Interactions. Biol. Psychiatry 2011, 69, 762-771. [CrossRef]

32. Eszlari, N.; Kovacs, D.; Petschner, P.; Pap, D.; Gonda, X.; Elliott, R.; Anderson, I.M.; Deakin, J.F.W.; Bagdy, G.; Juhasz, G. Distinct effects of folate pathway genes MTHFR and MTHFD1L on ruminative response style: A potential risk mechanism for depression. Transl. Psychiatry 2016, 6, e745. [CrossRef]

33. Johnson, D.P.; Rhee, S.H.; Friedman, N.P.; Corley, R.P.; Munn-Chernoff, M.A.; Hewitt, J.K.; Whisman, M.A. A Twin Study Examining Rumination as a Transdiagnostic Correlate of Psychopathology. Clin. Psychol. Sci. 2016, 4, 971-987. [CrossRef]

34. Chu, T.T.; Liu, Y. An integrated genomic analysis of gene-function correlation on schizophrenia susceptibility genes. J. Hum. Genet. 2010, 55, 285-292. [CrossRef]

35. Georgescu, I.A.; Popa, D.; Zagrean, L. The Anatomical and Functional Heterogeneity of the Mediodorsal Thalamus. Brain Sci. 2020, 10, 624. [CrossRef] [PubMed]

36. Mexal, S.; Berger, R.; Pearce, L.; Barton, A.; Logel, J.; Adams, C.E.; Ross, R.G.; Freedman, R.; Leonard, S. Regulation of a novel alphaN-catenin splice variant in schizophrenic smokers. Am. J. Med Genetics. Part B Neuropsychiatr. Genet. Off. Publ. Int. Soc. Psychiatr. Genet. 2008, 147, 759-768. [CrossRef] [PubMed]

37. Abe, K.; Chisaka, O.; Van Roy, F.; Takeichi, M. Stability of dendritic spines and synaptic contacts is controlled by alpha N-catenin. Nat. Neurosci. 2004, 7, 357-363. [CrossRef] [PubMed]

38. Park, C.; Falls, W.; Finger, J.H.; Longo-Guess, C.M.; Ackerman, S.L. Deletion in Catna2, encoding alpha N-catenin, causes cerebellar and hippocampal lamination defects and impaired startle modulation. Nat. Genet. 2002, 31, 279-284. [CrossRef]

39. Braff, D.L. Prepulse inhibition of the startle reflex: A window on the brain in schizophrenia. Curr. Top. Behav. Neurosci. 2010, 4, 349-371. [CrossRef]

40. Bagyura, Z.; Kiss, L.; Edes, E.; Lux, A.; Polgar, L.; Soos, P.; Szenczi, O.; Szelid, Z.; Vadas, R.; Jozan, P.; et al. Cardiovascular screening programme in the Central Hungarian region. The Budakalasz Study. Orv. Hetil. 2014, 155, 1344-1352. [CrossRef]

41. Nuttall, F.Q. Body Mass Index: Obesity, BMI, and Health: A Critical Review. Nutr. Today 2015, 50, 117-128. [CrossRef]

42. D'Agostino, R.B., Sr.; Vasan, R.S.; Pencina, M.J.; Wolf, P.A.; Cobain, M.; Massaro, J.M.; Kannel, W.B. General cardiovascular risk profile for use in primary care: The Framingham Heart Study. Circulation 2008, 117, 743-753. [CrossRef]

43. D'Agostino, R.B.; Wolf, P.A.; Belanger, A.J.; Kannel, W.B. Stroke risk profile: Adjustment for antihypertensive medication. The Framingham Study. Stroke 1994, 25, 40-43. [CrossRef] [PubMed]

44. Wilson, P.W.; D’Agostino, R.B.; Levy, D.; Belanger, A.M.; Silbershatz, H.; Kannel, W.B. Prediction of coronary heart disease using risk factor categories. Circulation 1998, 97, 1837-1847. [CrossRef] [PubMed]

45. Derogatis, L.R. BSI: Brief. Symptom Inventory: Administration, Scoring, and Procedures Manual; National Computer Systems Pearson, Inc.: Minneapolis, MN, USA, 1993.

46. Coleman, J.R.; Euesden, J.; Patel, H.; Folarin, A.A.; Newhouse, S.; Breen, G. Quality control, imputation and analysis of genomewide genotyping data from the Illumina HumanCoreExome microarray. Brief. Funct. Genom. 2016, 15, 298-304. [CrossRef] [PubMed]

47. Lippert, C.; Listgarten, J.; Liu, Y.; Kadie, C.M.; Davidson, R.I.; Heckerman, D. FaST linear mixed models for genome-wide association studies. Nat. Methods 2011, 8, 833-835. [CrossRef]

48. Gao, X.; Starmer, J.; Martin, E.R. A multiple testing correction method for genetic association studies using correlated single nucleotide polymorphisms. Genet. Epidemiol. 2008, 32, 361-369. [CrossRef] 
49. Gauderman, W.J. Sample size requirements for matched case-control studies of gene-environment interaction. Stat. Med. 2002, 21, 35-50. [CrossRef]

50. Draper, D. Bayesian multilevel analysis and MCMC. In Handbook of Multilevel Analysis; Springer: New York, NY, USA, 2008; pp. 77-139.

51. Antal, P.; Millinghoffer, A.; Hullám, G.; Szalai, C.; Falus, A. A Bayesian View of Challenges in Feature Selection: Feature Aggregation, Multiple Targets, Redundancy and Interaction. In Proceedings of the Workshop on New Challenges for Feature Selection in Data Mining and Knowledge Discovery at ECML/PKDD 2008, Antwerp, Belgium, 15 September 2008 ; pp. 74-89.

52. Cooper, G.F.; Herskovits, E. A Bayesian Method for the Induction of Probabilistic Networks from Data. Mach. Learn. 1992, 9, 309-347. [CrossRef] 